\title{
Parp1-Dependent DNA Double-Strand Break Repair in Irradiated Late Pachytene Spermatocytes
}

\author{
Emad A. Ahmed, ${ }^{1,2}$ Abdullah M. Alzahrani, ${ }^{1}$ and Harry Scherthan ${ }^{3}$
}

\begin{abstract}
Poly (ADP-ribose) polymerase-1 (Parp1) is a member of nuclear enzymes family involved in to the response to genotoxic stresses, DNA repair, and is critical for the maintenance of genome stability. During gametogenesis, genome stability is essential for inheritance and formation of healthy gametes. The latter involves DNA doublestrand break (DSB)-driven pairing of homologous chromosomes in first meiotic prophase. By analysis of DSB repair kinetics in male meiotic prophase cells of homologous recombination (HR) and nonhomologous end joining (NHEJ)-deficient mouse models, we previously demonstrated an interplay between HR and the conventional NHEJ repair pathway. In the current work, we evaluate the relative contribution of Parp1-dependent NHEJ to the repair of ectopic ionizing radiation (IR)-induced DSBs in control and Parp1-inhibited mouse pachytene spermatocytes before and after the completion of meiotic recombination in stages VI-XI. The disappearance of large, exogenous DSB-related $\gamma$-H2AX foci was quantified 1 and $8 \mathrm{~h}$ after 1 Gy $\gamma$-irradiation of control and 3,4-dihydro-5-[4-(1-piperidinyl)butoxy]-1(2H)quinolinone (DPQ) Parp1-inhibited mice. Late pachytene control spermatocytes obtained $8 \mathrm{~h}$ after IR had repaired $>80 \%$ of DSBs observed at $1 \mathrm{~h}$ after IR. However, only 64\% of DSBs were repaired in late spermatocytes of DPQ-treated (Parp1-inhibited) mice. Thus, it appears that Parp1 contributes to the repair of a fraction of DSBs in late prophase I, providing further insights in DNA repair pathway choreography during spermatogenic differentiation.
\end{abstract}

Keywords: alternative NHEJ, DNA repair, first meiotic prophase, $\gamma$-H2AX, homologous recombination, Parp1, spermatogenesis

\section{Introduction}

$\mathbf{T}$ he POly (ADP-Ribose) POlYMerase 1 (Parp1) enzyme is an abundant chromatin-associated protein that has a variety of cellular functions and is implicated in DNA damage signaling and repair, chromatin remodeling, and transcriptional regulation (Chaudhuri and Nussenzweig, 2017; Pazzaglia and Pioli, 2020). Parp1 is contributing to a number of nuclear and cellular functions including DNA repair (Thomas and Tulin, 2013; Chaudhuri and Nussenzweig, 2017; Slade, 2019). Besides being required for base excision repair and its role in other DNA repair pathways, Parp1 is also involved in the alternative nonhomologous end joining (aNHEJ) repair pathway. While some details are still not clear, aNHEJ seems to utilize the synaptic activity of Parp1 and the ligation activity of the XRCC1-DNA ligase III complex (Soni et al., 2014; Kang and Yan, 2018). This alternative pathway of NHEJ can function as a backup to the DNA-PK-dependent classical NHEJ (cNHEJ) pathway, particularly in the absence of $\mathrm{Ku}$ or more essential com- ponents of the cNHEJ pathway or when Parp1 and $\mathrm{Ku}$ compete for repair of double-strand breaks (DSBs) (Mansour et al., 2013; Bakr et al., 2016). Although Parp1knockout mice are viable and fertile with normal life span, they show altered inflammatory processes and are more sensitive to ionizing radiation (IR) and show high levels of chromatid and chromosome aberrations after exposure to genotoxic agents (de Murcia et al., 1997; Wang et al., 1997; Shall and de Murcia, 2000).

Upon IR-induced DSB formation, the histone variant $\mathrm{H} 2 \mathrm{AX}$ is phosphorylated at serine 139 in the chromatin surrounding a DSB (Rogakou et al., 1998), leading to microscopically visible DSB-indicating $\gamma$-H2AX nuclear foci (Rogakou et al., 1999; Iacovoni et al., 2010). $\gamma$-H2AX focus formation and disappearance are widely used as markers reflecting the extent of DSB damage and repair (Rothkamm et al., 2015; Ahmed et al., 2017; Singh et al., 2018). It is of note that a $\gamma$-H2AX focus may contain more than one DSB at higher radiation doses (Scherthan et al., 2008; Antonelli et al., 2015).

\footnotetext{
${ }^{1}$ Biological Sciences Department, Faculty of Science, King Faisal University, Al-Ahsa, Saudi Arabia.

${ }^{2}$ Laboratory of Molecular Physiology, Zoology Department, Faculty of Science, Assiut University, Assiut, Egypt.

${ }^{3}$ Institut für Radiobiologie der Bundeswehr in Verb. mit der Universität Ulm, Munich, Germany.
} 
In male germ cells, at leptotene substage of first meiotic prophase, endogenous DSBs are introduced by SPO11, a topoisomerase II-like endonuclease (Keeney et al., 1997; Baudat et al., 2000). These physiologically induced DSBs contribute to homologous chromosome recognition and are repaired through homologous recombination (HR), which is a highly orchestrated process (Hunter, 2015) that depends on homologous chromosome pairing and synapsis, established during zygotene stage (for review, see Zickler and Kleckner, 2015). Crossovers are formed at least once between each pair of homologous chromosomes during the pachytene stage (Gerton and Hawley, 2005). Two types of DSBdependent $\gamma$-H2AX chromatin foci have been described in spermatocyte nuclei at pachytene stage of meiosis: small $\gamma$ $\mathrm{H} 2 \mathrm{AX}$ foci that mark the Spo11-related DSBs sites and are located mostly at the synaptonemal complex (SC) and larger $\gamma$-H2AX foci (signals) encompassing chromatin loops emanating from the SC that are induced by both unrepaired Spo11-DSBs and IR-induced exogenous DSBs (Chicheportiche et al., 2007; Ahmed et al., 2018).

Previous analysis of Parp1 expression level in the testes revealed a higher expression in primary spermatocytes relative to other male germ cells (Di Meglio et al., 2003). However, based on its staining intensity, Parp1 expression appears to increase gradually from early to late meiotic prophase spermatocytes (Ahmed et al., 2010b). However, the lack of Parp1 function during oogenesis was found to predispose the female gamete to genome instability and consequently to a spectrum of meiotic defects including incomplete homologous chromosome synapsis or persistent $\gamma$-H2AX foci along fully synapsed chromosomes at the late female pachytene stage (Yang et al., 2009). In male severe combined immunodeficiency (SCID) mice, an elevated number of apoptotic pachytene spermatocytes were detected at the stage IV checkpoint of the testicular epithelial cycle, which has been attributed to a role of the cNHEJ pathway during the repair of meiotic DSBs (de Rooij and de Boer, 2003; Hamer et al., 2003). However, at variance, no difference was found in the repair kinetics of IR-induced DSBs in early pachytene spermatocytes from SCID and control mice (Ahmed et al., 2019). In addition, $\mathrm{Ku}$ proteins that contribute to cNHEJ are not expressed during early meiotic prophase of primary wild-type spermatocytes (Ahmed et al., 2013). However, investigation of repair kinetics in late spermatocytes of cNHEJ-deficient SCID mice has revealed that that about $11 \%$ of IR-induced DSBs are not repaired relative to control, indicating that $\mathrm{cNHEJ}$ plays a role in the repair of a subset of DSBs in late spermatocytes (Ahmed et al., 2017). Furthermore, Parp1/XRCC1-dependent aNHEJ is active in postmeiotic spermatids (Ahmed et al., 2010a). Thus, the understanding of processes contributing to the repair of exogenous DSB damage during and after completion of meiotic recombination requires more analyses.

Given the higher expression of Parp1 in late spermatocytes (Maymon et al., 2006; Tramontano et al., 2007; Ahmed et al., 2010b), the declining activity of HR to repair DSBs in late pachytene, and the possible role for cNHEJ in repairing a subset of DSBs in these cells, there remains the question whether Parp1-dependent aNHEJ also contributes to this DSB damage repair in late pachytene and diplotene spermatocytes. Here, we addressed this question evaluating the relative contribution of the Parp1-dependent end joining repair pathway in repairing exogenous irradiation DSBs in late prophase I spermatocytes.

\section{Materials and Methods}

\section{Animals and irradiation}

The effect of the Parp1 inhibitor 3,4-dihydro-5-[4-(1piperidinyl)butoxy]-1(2H)quinolinone (DPQ) on Parp1dependent damage repair was studied in male, 8- to 10week-old BALB/c albino mice, a similar model to our previous investigations (Ahmed et al., 2010). Mice were bred at the animal house of Zoology Department, Faculty of Science, Assiut University. Animal experiments were done according to animal welfare rules stated by Assiut University and King Faisal University (KFU/REC 2020/02/07, approved February, 2020). In total, 24 male mice were used in the experiment. Mice were maintained under standard laboratory conditions at ambient temperature $\left(25^{\circ} \mathrm{C}\right)$, relative humidity $60 \%-70 \%$, and a $12 \mathrm{~h}$ light/dark cycle. They were allowed to acclimatize for 1 week ahead of the experiment. Mice were fed a standard commercial pellet diet and water and housed in polypropylene cages inside a well-ventilated room.

In brief, mice were intraperitoneally injected with the Parp1 inhibitor DPQ (D5314; Sigma-Aldrich) dissolved in dimethyl sulfoxide (DMSO) or DMSO alone at $10 \mathrm{mg} / \mathrm{kg}$ of body weight. Two injections were applied to inhibit Parp1: the first DPQ injection was given $1.5 \mathrm{~h}$ before $1 \mathrm{~Gy}$ gamma ray whole-body irradiation with a gamma ray source (Elekta, Sweden) at $0.8 \mathrm{~Gy} / \mathrm{min}$ dose rate. The second injection was given $3.5 \mathrm{~h}$ after irradiation. This protocol was followed since it has been useful for DNA repair studies in the mouse testes (Ahmed et al., 2010a). After 1 and $8 \mathrm{~h}$ of whole body exposure to a dose of $1 \mathrm{~Gy} 6 \mathrm{MV}$ gamma rays (four mice per group), mice were sacrificed and one testis was used to prepare meiotic spreads (Muhtadi et al., 2017; Ahmed et al., 2018) and the other was fixed in $10 \%$ formalin for $16 \mathrm{~h}$, followed by paraffin embedding for immunohistochemical investigation.

\section{Immunohistochemistry}

Paraffin sections $(6 \mu \mathrm{m})$ of testes of control and irradiated mice were mounted together on SuperFrost glass slides and dried overnight at $37^{\circ} \mathrm{C}$. Sections were dewaxed in xylene, followed by hydration in a graded series of alcohols. The sections were boiled once (each for $10 \mathrm{~min}$ ) in $0.01 \mathrm{M}$ sodium citrate $(\mathrm{pH}=6)$ using a microwave oven (Bio- $\mathrm{Rad})$ and incubated in $0.35 \% \mathrm{H}_{2} \mathrm{O}_{2}$ in phosphate-buffered saline (PBS) for $10 \mathrm{~min}$. Unspecific protein binding was blocked by $5 \mathrm{~min}$ incubation in 5\% bovine serum albumin (Sigma-Aldrich) and 5\% goat serum in PBS. The slides were incubated with the primary antibody (anti- $\gamma$-H2AX; Upstate; and anti-Parp1; Abcam; see the Antibodies section) for $1 \mathrm{~h}$ at room temperature, followed by washing in PBS and then incubation with the ready-to-use secondary antibody PowerVision poly-anti-mouse/rabbit-HRP (ImmunoVision). Bound antibodies were visualized using $0.3 \mu \mathrm{g}$ 3,3-diaminobezidine (Sigma-Aldrich) in PBS, to which $0.03 \% \mathrm{H}_{2} \mathrm{O}_{2}$ was added. Sections were counterstained with Mayer's hematoxylin (Sigma-Aldrich).

\section{Surface spread preparations}

Nuclear spreads of spermatocytes were prepared as previously described (Peters et al., 1998; Ahmed et al., 2010; 
Muhtadi et al., 2017). Briefly, a suspension of testis cells was prepared by mincing in MEM- $\alpha$, followed by a $10-\mathrm{min}$ incubation with a hypotonic buffer $(17 \mathrm{mM}$ sodium citrate, $50 \mathrm{mM}$ sucrose, $30 \mathrm{mM}$ Tris- $\mathrm{HCl}, \mathrm{pH} 8.2)$. After being centrifuged, the pellet was carefully resuspended in a $100 \mathrm{mM}$ sucrose solution and applied over a paraformaldehyde (PFA)covered glass slide (1\% PFA, $0.15 \%$ Triton-X-100, pH 9.29.5). Slides were kept in a humidified atmosphere in a box for slow dying down. After $1.5 \mathrm{~h}$, the box was opened and the slides were washed in $0.08 \%$ photo-flo (Sigma-Aldrich).

\section{Antibodies}

The primary antibodies were rabbit polyclonal anti-SCP3 (1:400, ab15092; Abcam), mouse monoclonal anti-phosphoH2AX [Ser139] (1:400, 16-202A; Upstate Biotechnology), and rabbit anti-Parp1 (1:100, Abcam). The secondary antibodies, goat anti-rabbit (Alexa Fluor 488, A-11008) and Texas Red-labeled goat anti-mouse (T6134), were obtained from Jackson ImmunoResearch and applied at a 1:1000 dilution.

\section{Fluorescent immunostaining and analysis}

For immunofluorescent staining, preparations were washed in PBS and incubated for $10 \mathrm{~min}$ in PBS, $0.04 \%$ Triton$\mathrm{X}-100$, followed by incubation in blocking solution $(10 \%$ BSA in PBS for $30 \mathrm{~min}$.). After blocking, primary antibodies (see the Antibodies section) were applied, diluted in blocking solution, for $2 \mathrm{~h}$ at room temperature. Then, slides were washed in PBS and incubated with the diluted secondary antibodies (see the Antibodies section) in blocking solution for $1 \mathrm{~h}$ at room temperature. Thereafter, cell nuclei were stained with DAPI for $10 \mathrm{~min}$ (concentration in $\mathrm{H}_{2} \mathrm{O}$ ) and mounted in VECTAshield antifade solution (Vector Labs) until inspection.

For immunohistochemistry (IHC) staining, the analysis was carried out in the spermatocytes after identifying epithelial stage according to the method described in detail by Ahmed and de Rooij (2009). Spermatocyte signal intensity measurements were performed using the TissueQuest software (TissueGnostics, Vienna). In brief, the outline of spermatocyte nuclei (blue, DAPI) was determined by the software based on DAPI staining and gray value drop-offs and size of nuclei. Inside the nuclear masks, fluorescence intensities of the green channel were computed for each nucleus and presented as average and standard deviation (SD) of arbitrary gray value units.

To analyze the relative contribution of Parp1-dependent pathway in the repair of exogenously induced DSBs during late prophase $\mathrm{I}$, we studied the kinetics of irradiationinduced large DSB-indicating " $L " ~ \gamma-\mathrm{H} 2 \mathrm{AX}$ foci that emanate from the SC in nuclear spreads as described previously (Chicheportiche et al., 2007; Ahmed et al., 2018). Foci disappearance was studied of control and DPQ-treated mice to determine repair potency. Foci numbers were also scored in pachytene spermatocytes from paraffin-embedded testis sections at defined testicular epithelial stages as described earlier (Ahmed and de Rooij, 2009).

Preparations were viewed with an Olympus epifluorescence microscope (Olympus Tokyo, Japan). Digital gray scale images were recorded using a $63 \times$ lens and a digital 20MP camera (Leica) and merged using the ImageJ software. Focus counting was performed on the merged RGB images and foci were enumerated in up to 100 wellstained cells from three to four mice per group.

\section{Statistical analysis}

The data were analyzed using Student's $t$-test using QuickCalcs software (graphpad.com), and the data are expressed as mean \pm standard error of the mean.

\section{Results}

\section{$D P Q$ treatment reduces Parp1-staining intensity in irradiated late spermatocytes}

In wild-type mice, Parp1 is expressed in all germ cell types with low staining intensity in early meiotic prophase cells, that is, preleptotene, leptotene, and zygotene spermatocytes (Ahmed et al., 2010b). Nonirradiated control and DPQ-treated (Parp1-inhibited) testes cells failed to display significant differences in Parp1 staining intensity (unpublished observations). However, ionizing radiation (IR) of control mice induced an increase of Parp1 staining intensity in late spermatocytes (late pachytene), whereas irradiated DPQ-treated mice showed a significant reduction in Parp1 staining intensity (Supplementary Fig. S1). As expected, IR exposure also induced DSB-indicating $\gamma$-H2AX foci numbers in spread spermatocytes (Fig. 1A-F) and spermatocytes of tissue sections of control and Parp1-inhibited mice (Fig. 1G-L).

\section{Parp1-dependent DNA repair in early pachytene spermatocytes}

Next, we determined the average $\gamma$-H2AX foci number per spermatocyte (surrogate marker for DSBs) (Mahadevaiah et al., 2001; Chicheportiche et al., 2007; Lobrich et al., 2010; Singh et al., 2018) in testicular sections of irradiated control and Parp1-inhibited mice in situ after $\gamma$ H2AX IHC staining (Figs. 1G-L and 2A-F). The kinetics of DSB repair was derived from $\gamma-\mathrm{H} 2 \mathrm{AX}$ focus formation and disappearance (Ahmed et al., 2018; Singh et al., 2018) between 1 and $8 \mathrm{~h}$ after irradiation in seminiferous epithelial stages I-III, IV-V, VI-IX, and X-XI.

Irradiated early to mid-pachytene spermatocytes of stages $\mathrm{I}-\mathrm{V}$ testis tubules displayed similar average DSB-indicating $\gamma-\mathrm{H} 2 \mathrm{AX}$ foci numbers in control and DPQ-treated mice (Figs. 2G and 3A). This suggests that Parp1 plays no role in the repair of endogenous and exogenous dsDNA damage in early prophase I spermatocytes up to the mid-pachytene stage, agreeing with the view that HR being the major repair pathway in the early prophase I substages.

\section{Reduced DNA repair in late pachytene spermatocytes of Parp1-inhibited mice}

The investigation of $\gamma-\mathrm{H} 2 \mathrm{AX}$ DSB foci in irradiated late pachytene spermatocytes after completion of crossing over, that is, in seminiferous stages VI-XI, revealed a Parp1 inhibition-dependent significant increase in the average number of $\gamma$-H2AX DSB foci/spermatocyte $1 \mathrm{~h}$ after IR exposure relative to the control (Fig. 3A), indicating that Parp1/Parp1-dependent aNHEJ is contributing to fast DNA repair. Likewise, Parp1-inhibited late pachytene spermatocytes of epithelial stages VI-VIII and IX-XI $8 \mathrm{~h}$ after IR also displayed a significant increase $(p<0.01)$ in the average numbers of persistent foci $(3.7 \pm 0.16$ foci per cell [fpc]) in stages VI-VII late pachytene spermatocytes 

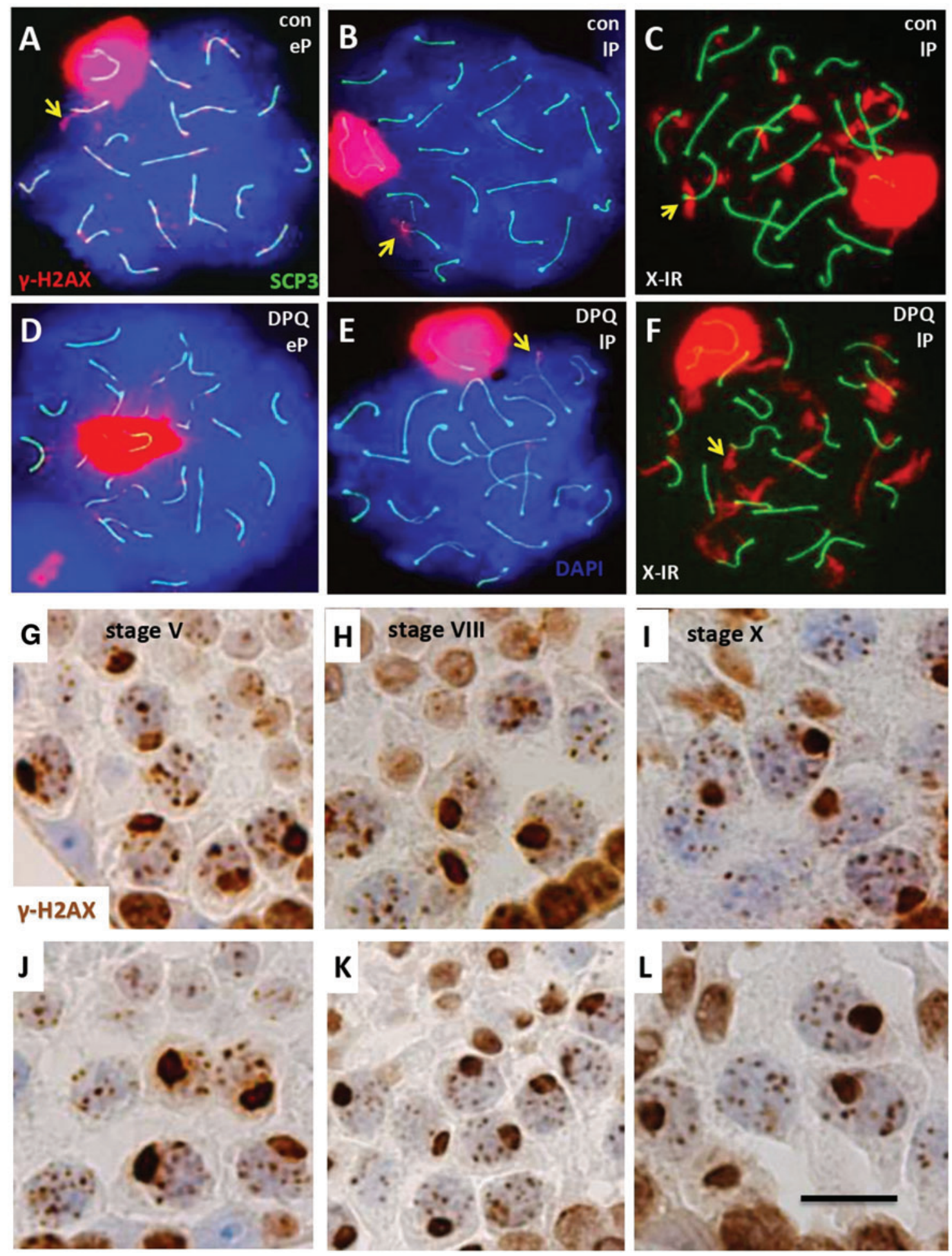

FIG. 1. Meiotic recombination-related $\gamma-\mathrm{H} 2 \mathrm{AX}$ foci in spread pachytene spermatocytes of control (con; A-C) and DPQtreated mice (DPQ; D-F). eP spermatocytes show numerous $\gamma-\mathrm{H} 2 \mathrm{AX}$ (red) foci in their chromatin (A, D). Persistent spontaneous large (L) $\gamma$-H2AX foci (examples arrowed) in contact with the SC (green) of IP spermatocytes (B, E). Numerous foci are observed in meiotic spreads of 1 Gy irradiated of IP spermatocytes of control (C) and DPQ-treated mice (F) $1 \mathrm{~h}$ after $\gamma$-IR. DNA was counterstained DAPI (blue). Representative images of testes tissue sections (G-L; different epithelial stages are shown) $1 \mathrm{~h}$ after $\gamma$-irradiation stained for DSB marker $\gamma$-H2AX (DAB, brown) by IHC of control (G-I) and DPQ-treated mice (J-L). Sex chromosomes (XY body) show strong $\gamma-\mathrm{H} 2 \mathrm{AX}$ fluorescence (red). Magnification bar: $10 \mu \mathrm{m}$. DAB, 3,3-diaminobezidine; DPQ, 3,4-dihydro-5-[4-(1-piperidinyl)butoxy]-1(2H)quinolinone; DSB, double-strand break; eP, early pachytene; IHC, immunohistochemistry; IR, ionizing radiation; IP, late pachytene; SC, synaptonemal complex. Color images are available online. 

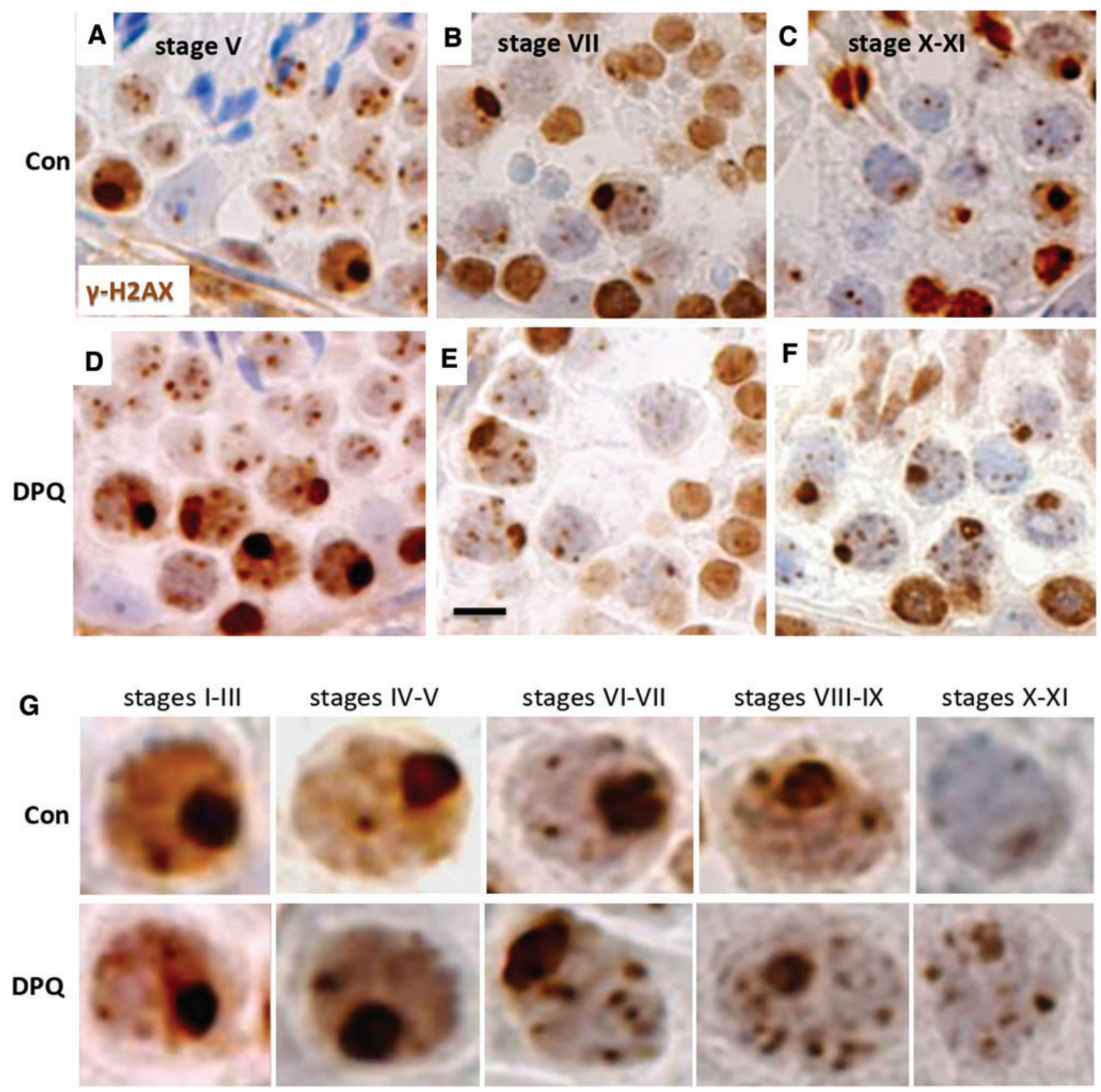

FIG. 2. $\gamma$-H2AX staining of testicular sections of $1 \mathrm{~Gy} \gamma$-irradiated control (A-C) and DPQ-treated mice (D-F) $8 \mathrm{~h}$ after IR. Representative images from various epithelial stages showing $\gamma-\mathrm{H} 2 \mathrm{AX}$ foci (dark brown; DAB IHC) and XY bodies (large dark blob) in spermatocytes of stages IV-V (A, D), VI-VII (B, E), and X-XI (C, F). Representative images of individual pachytene spermatocytes (G) with XY body (large dark area) and DSB foci from different epithelial stages of control (Con) and DPQ-treated mice as indicated, $8 \mathrm{~h}$ after irradiation. Color images are available online.

(1P) and $5.1 \pm 0.15 \mathrm{fpc}$ in stages IX-XI $1 \mathrm{P}$, respectively, while control stages VI-VIII IP displayed $2.3 \pm 0.12 \mathrm{fpc}$ and $3.1 \pm 0.12 \mathrm{fpc}$ in $\mathrm{lP}$ of stage IX-XI tubules.

The kinetics of foci disappearance (repair) between 1 and $8 \mathrm{~h}$ was significantly $(p=0.04)$ reduced in stages VI-VIII IP spermatocytes from $78 \%$ in control to $\sim 66 \%$ in Parp1inhibited mice (Fig. 3C). In stages IX-XI 1P, the average number of large $\gamma-\mathrm{H} 2 \mathrm{AX}$ foci was reduced from $68.3 \%$ in control to 55.4\% in Parp1-inhibited mice (Fig. 3C). This reduction is significant $(p=0.004)$, indicating that in late pachytene spermatocytes Parp1 contributes to the repair of about $10 \%$ of exogenously (IR) induced DSB foci during $8 \mathrm{~h}$ following a genotoxic insult.

\section{Parp1-dependent DNA repair in spread late pachytene spermatocytes}

To determine and control the observed DSB numbers at a higher resolution, we next performed nuclear spreads of spermatocytes (Muhtadi et al., 2017) and investigated the 
FIG. 3. DNA repair kinetics in spermatocytes from different epithelial stages of irradiated control and DPQ-treated mice 1 and $8 \mathrm{~h}$ after IR. (A) $\gamma$-H2AX foci numbers $1 \mathrm{~h}$ after IR in different epithelial stages. While pachytene nuclei in stages IV-V tubuli showed similar average $\gamma-\mathrm{H} 2 \mathrm{AX}$ foci numbers per cell, these were significantly increased in stages VI-XI, indicating a contribution of aNHEJ in DSB repair late in prophase I. (B) Parp1-inhibited spermatocytes show significantly more induced $\gamma$ $\mathrm{H} 2 \mathrm{AX}$ foci numbers $8 \mathrm{~h}$ after $\gamma$-irradiation. (C) Percentages of DSB repair in pachytene spermatocytes of indicated testicular epithelial stages $8 \mathrm{~h}$ after IR relative to $1 \mathrm{~h}$ after IR. The data shown are derived from up to 100 cells from three mice each; a, significant difference to control at $* p \geq 0.04$ and $* * p \geq 0.01$ ( $t$-test). Error bars represent SEM. aNHEJ, alternative nonhomologous end joining; Parp1, poly (ADP-ribose) polymerase-1; SEM, standard error of the mean.
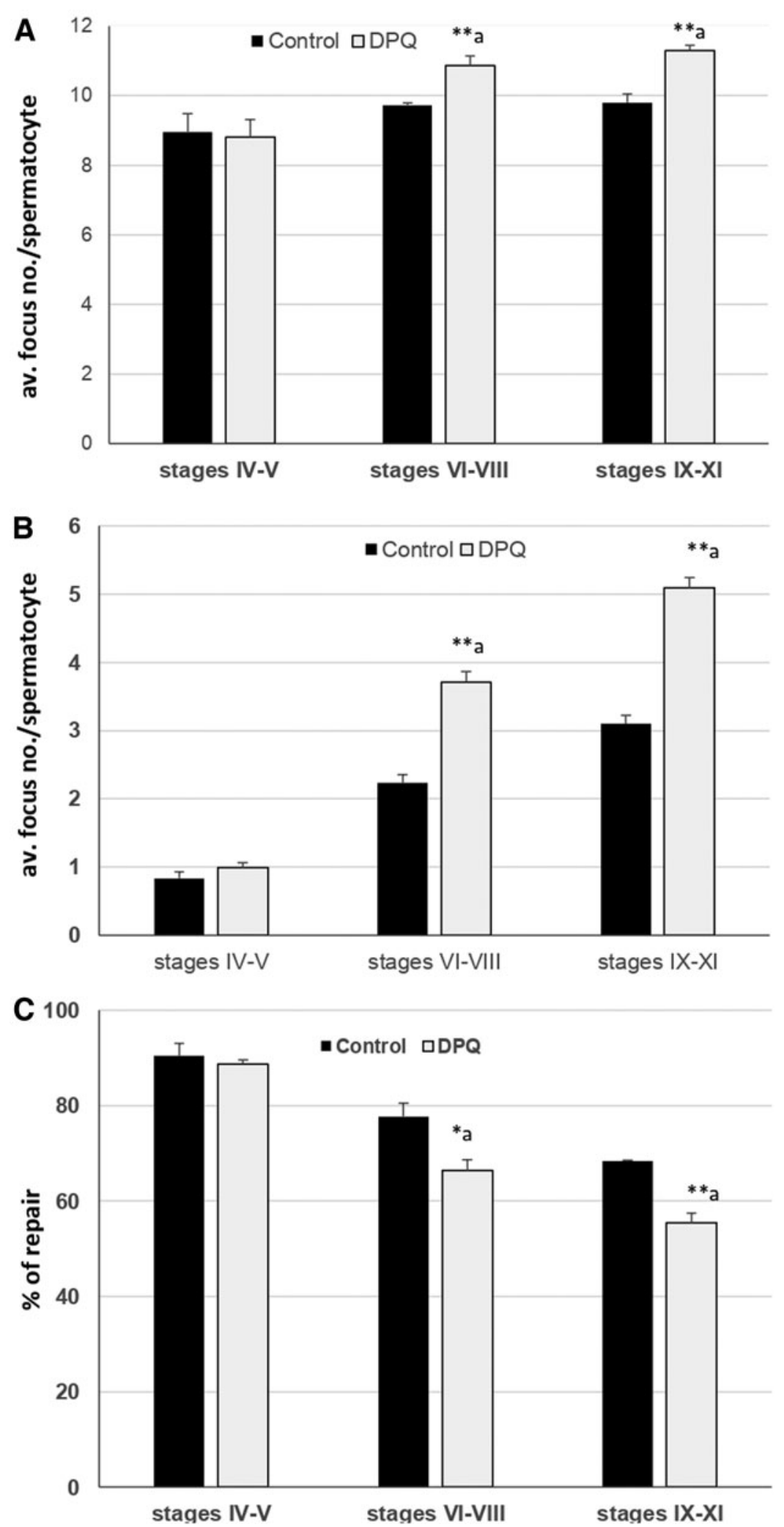

DSB repair kinetics in both groups by enumeration of $\mathrm{L}-\gamma$ $\mathrm{H} 2 \mathrm{AX}$-foci (Fig. 4A-F) that are a result of DSBs in association with the SC (Chicheportiche et al., 2007; Ahmed et al., 2018). The significant decline of the average $\gamma-\mathrm{H} 2 \mathrm{AX}$ foci numbers in tissue sections of DPQ-inhibited sper- matocytes relative to control was also detected in $\gamma-\mathrm{H} 2 \mathrm{AX}$ stained meiotic nuclear spreads (Figs. 1A-F and 4A-F), where the numbers of persistent $\mathrm{L}-\gamma-\mathrm{H} 2 \mathrm{AX}$ foci in Parp1inhibited late spermatocytes (Fig. 4E, F) $(6.4 \pm 0.28 \mathrm{fpc})$ were almost twofold relative to untreated control (Fig. 4B, C) 

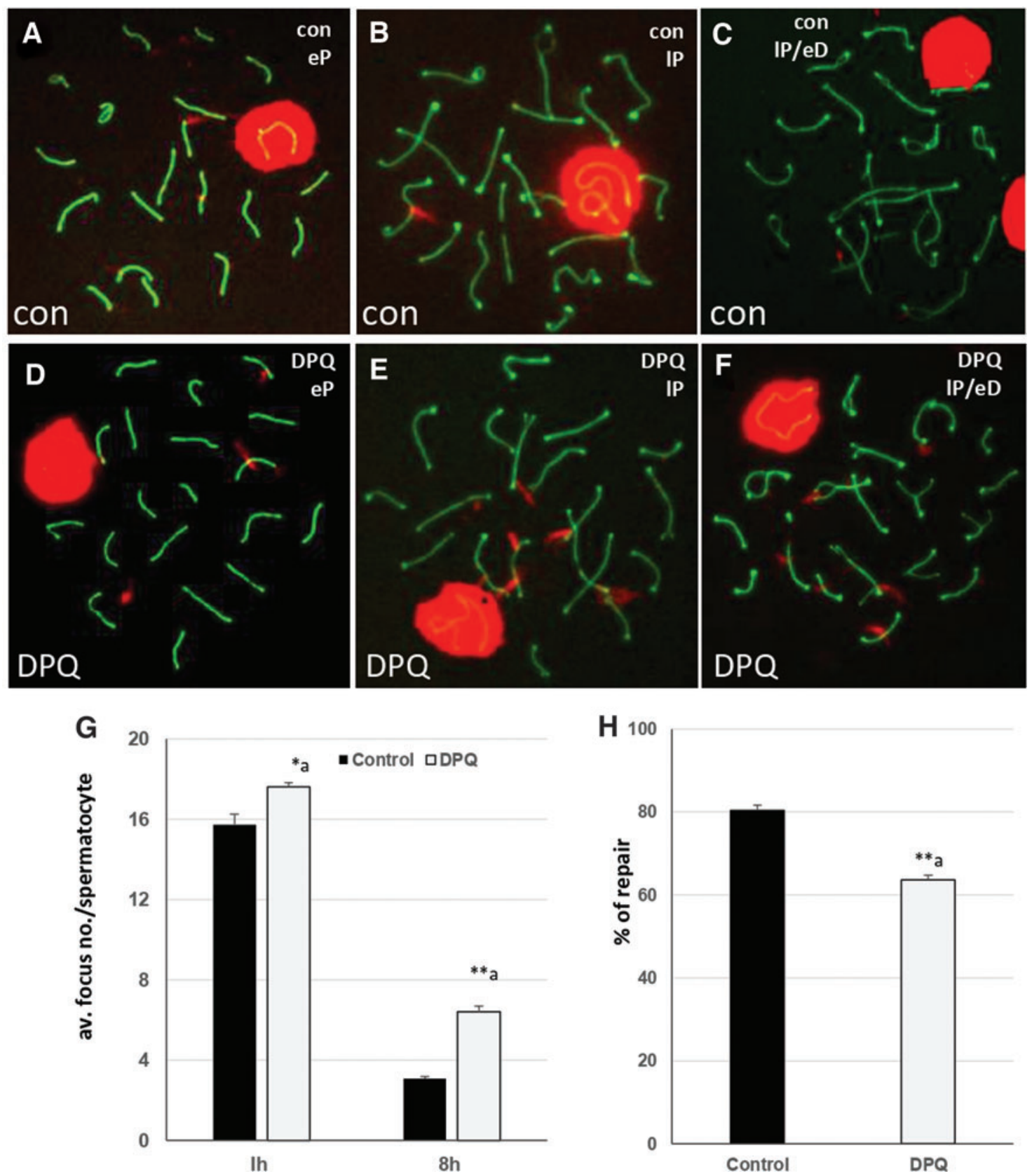

FIG. 4. DNA repair kinetics in nuclear spreads of irradiated pachytene spermatocytes of control (A-C) and DPQ-treated (D-F) mice $8 \mathrm{~h}$ after IR. eP $(\mathbf{A}, \mathbf{D}), \mathrm{lP}(\mathbf{B}, \mathbf{E})$, and IP/eD $(\mathbf{C}, \mathbf{F})$ spermatocytes as indicated. L- $\gamma$-H2AX foci numbers are significantly increased in DPQ-treated mice $(\mathbf{G})$ indicating a significantly reduced repair efficiency between 1 and $8 \mathrm{~h}$ after IR (H). Sixty cells were counted from three mice per group; a, significant difference to control at $* p \geq 0.025$ and $* * p \geq 0.01$ ( $t$-test). eD, early diplotene. Color images are available online.

$(3.1 \pm 0.1)$ at $8 \mathrm{~h}$ after IR (Fig. 4G). This indicates a highly significant reduction $(p=0.0006)$ of the efficacy of DSB repair from $80 \%$ in control to $64 \%$ in the Parp1-inhibited late pachytene spermatocytes (Fig. 4H). Altogether, this again supports the conclusion of our tissue analysis above that Parp1 is required to repair a subset of exogenous DSBs in late prophase I spermatocytes.

\section{Discussion}

In this study, we have analyzed the contribution of Parp1 to dsDNA damage repair efficacy before and after comple- tion of crossing over in male first meiotic prophase at the boundary of mid to late pachytene substage after irradiation of control and Parp1-inhibited mouse testes. This approach allowed estimating the relative contribution of the Parp1dependent aNHEJ repair pathway to the repair of IR-induced exogenous DSBs in these germ cells. Meiotic prophase spermatocytes up to the mid-pachytene substage observed in seminiferous epithelial stages I-V (Ahmed and de Rooij, 2009) failed to display significant differences of $\gamma$-H2AXmarked exogenous DSB foci in control and DPQ treatedinhibited mice. Previous analysis of DSB repair kinetics in irradiated $\operatorname{Rad} 54 / \mathrm{Rad}_{54 B^{-/}}$HR-deficient mice revealed a 
significant reduction of about $37 \%$ of DSB repair relative to the irradiated wild type (Ahmed et al., 2010b). Therefore, the HR repair pathway that is predominant during early meiotic prophase also efficiently deals with exogenously induced DSBs. The latter may under certain conditions, for example, lack of endogenous DSBs, even function as substitute for HR hot spots (Liebe et al., 2006; Derijck et al., 2008). Our results, given the limitations of the assay used, suggest that cNHEJ and Parp1-dependent aNHEJ DSB repair pathways are dispensable for repairing endogenous and exogenous breaks in early prophase I spermatocytes before the mid-pachytene stage, where DSB repair is conducted mainly through HR (Ahmed et al., 2010b; Hunter, 2015).

Parp1 inhibition in mouse spermatogenesis by the potent inhibitor DPQ reduced the staining intensity of Parp1 in late pachytene spermatocytes, the stage when crossing over has been completed. At this stage, $1 \mathrm{~h}$ after irradiation, the number of $\gamma-\mathrm{H} 2 \mathrm{AX}$ DSB-indicating foci in Parp1-inhibited late pachytene spermatocytes was significantly elevated by $\sim 10 \%$ relative to the control. Previous studies in somatic and germ cells indicated that the absence of the fast cNHEJ repair pathway increased the number of unrepaired breaks at $1 \mathrm{~h}$ after IR (Iliakis et al., 2004; Ahmed et al., 2017). Using the same model, irradiated SCID late spermatocytes showed around $30 \%$ more DSB foci relative to those of control after $1 \mathrm{~h}$ of IR
(Ahmed et al., 2010). In the current model, we have observed a $10 \%$ increase in number of foci in Parp1-inhibited spermatocytes at $1 \mathrm{~h}$ after IR, which was significantly higher relative to control. Thus, Parp1 activity may, to some extent, contribute to the fast repair of DSBs in late prophase I spermatocytes, such as cNHEJ components do (Fig. 5) (Ahmed et al., 2017).

In late (after mid-pachytene) spermatocytes, the role of HR is declining (Fig. 5), as suggested by a similar DSB foci loss in HR-deficient and wild-type mice (Ahmed et al., 2010b). Data from cNHEJ-deficient SCID mice suggest that this pathway is responsible for the repair of about $30 \%$ of DSBs within the first hours after IR (Ahmed et al., 2010b). Earlier studies have also demonstrated the existence of an interplay between $\mathrm{HR}$ and cNHEJ in somatic cells (Fukushima et al., 2001; Allen et al., 2002; Kakarougkas and Jeggo, 2014; Bosshard et al., 2019), and a similar interplay has been suggested in the mouse zygote (Derijck et al., 2008). Here, we obtained data that suggest that in addition to the cNHEJ pathway the Parp1-dependent alternative NHEJ pathway contributes to the repair of a subset of exogenous IR-induced DSBs in late pachytene spermatocytes (Fig. 5) that show a declining HR repair activity. Since Parp1 inhibitor treatment seems to affect DNA repair through indirect effects (Flohr et al., 2003; Ma et al., 2012), it may be possible that the observed Parp1 contribution to

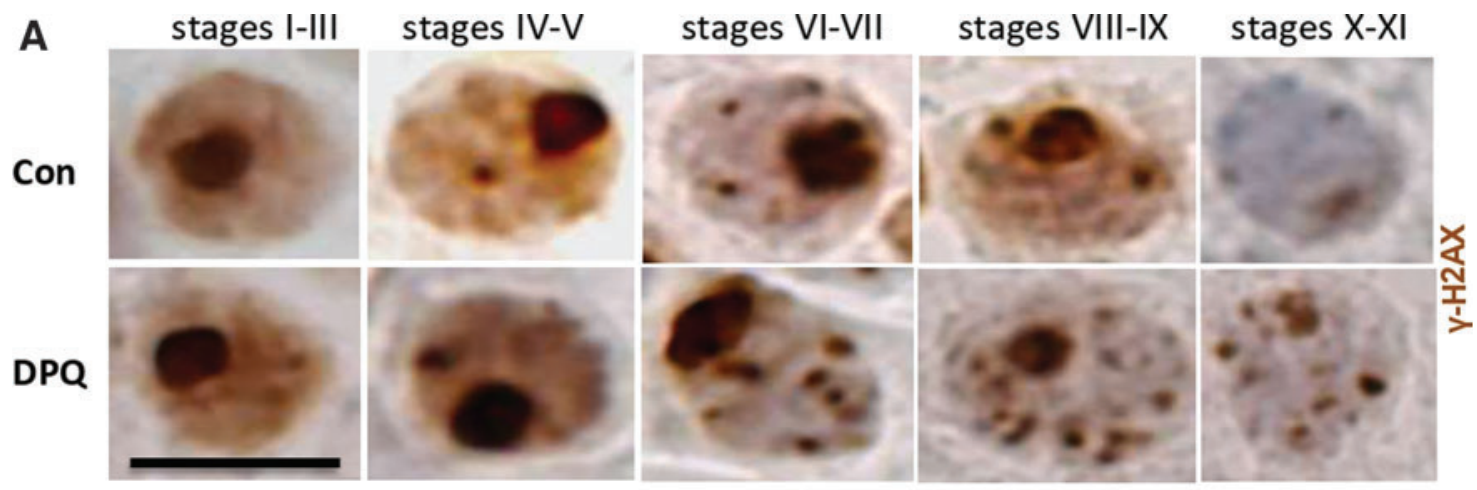

B

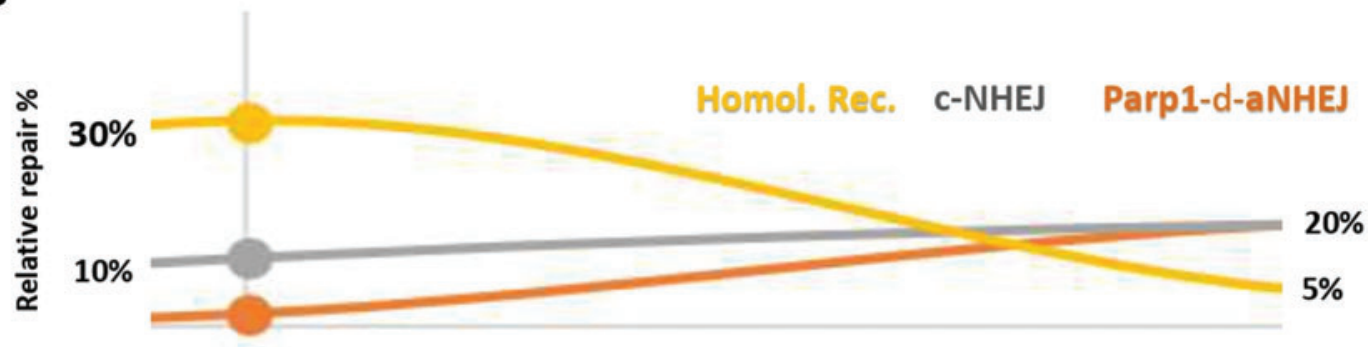

early pachytene

late pachytene

\section{Meiotic DSB repair}

FIG. 5. Outline of Parp1-dependent DSB repair during early and late pachytene of mouse meiotic prophase I with respect to NHEJ and HR repair. (A) Representative images of pachytene spermatocytes of testicular epithelial stages indicated stained for $\gamma-\mathrm{H} 2 \mathrm{AX}$ DSB damage (small dark brown foci) from mice with (DPQ) and without (Con) Parp1 inhibition. (B) Flowchart showing the percentage of relative DSB repair contribution by the respective pathways in late pachytene substage spermatocytes exposed to toxic DSBs. HR, homologous recombination. Color images are available online. 
aNHEJ repair of IR-induced DSBs in the G2-equivalent late prophase I spermatocytes could be indirect through its chromatin remodeling activity (Luijsterburg et al., 2016) and/or Parp1 influence on BER and other pathways. Thus, further analyses on the interplay of the different DNA repair pathways and the repair of toxic DSBs in late prophase I chromatin are required.

\section{Authors' Contributions}

E.A.A. designed the experiment, conducted practical experiments, analyzed data, prepared figures, and wrote the article. A.M.A. analyzed data, prepared figures, and revised the article. H.S. analyzed data, prepared figures, and revised the article.

\section{Acknowledgment}

H.S. thanks M. Port, Munich, for continuous support.

\section{Disclosure Statement}

No competing financial interests exist.

\section{Funding Information}

This study was supported by the Deanship of Scientific Research of King Faisal University for providing research group Grant No. 1811010, 2019.

\section{Supplementary Material}

Supplementary Figure S1

\section{References}

Ahmed, E.A., and de Rooij, D.G. (2009). Staging of mouse seminiferous tubule cross-sections. Methods Mol Biol 558, 263-277.

Ahmed, E.A., de Boer, P., Philippens, M.E., Kal, H.B., and de Rooij, D.G. (2010a). Parp1-XRCC1 and the repair of DNA double strand breaks in mouse round spermatids. Mutat Res 683, 84-90.

Ahmed, E.A., Philippens, M.E., Kal, H.B., de Rooij, D.G., and de Boer, P. (2010b). Genetic probing of homologous recombination and non-homologous end joining during meiotic prophase in irradiated mouse spermatocytes. Mutat Res 688, 12-18.

Ahmed, E.A., Rosemann, M., and Scherthan, H. (2018). NHEJ contributes to the fast repair of radiation-induced DNA double-strand breaks at late prophase I telomeres. Health Phy 115, 102-107.

Ahmed, E.A., Sfeir, A., Takai, H., and Scherthan, H. (2013). $\mathrm{Ku} 70$ and non-homologous end joining protect testicular cells from DNA damage. J Cell Sci 126, 3095-3104.

Ahmed, E.A., Velaz, E., Rosemann, M., Gilbertz, K.P., and Scherthan, H. (2017). DNA repair kinetics in SCID mice Sertoli cells and DNA-PKcs-deficient mouse embryonic fibroblasts. Chromosoma 126, 287-298.

Allen, C., Kurimasa, A., Brenneman, M.A., Chen, D.J., and Nickoloff, J.A. (2002). DNA-dependent protein kinase suppresses double-strand break-induced and spontaneous homologous recombination. Proc Natl Acad Sci U S A 99, 3758-3763.

Antonelli, F., Campa, A., Esposito, G., Giardullo, P., Belli, M., Dini, V., et al. (2015). Induction and repair of DNA DSB as revealed by $\mathrm{H} 2 \mathrm{AX}$ phosphorylation foci in human fibroblasts exposed to low- and high-LET radiation: relationship with early and delayed reproductive cell death. Radiat Res 183, 417-431.

Bakr, A., Köcher, S., Volquardsen, J., Petersen, C., Borgmann, K., Dikomey, E., et al. (2016). Impaired 53BP1/RIF1 DSB mediated end-protection stimulates CtIP-dependent end resection and switches the repair to PARP1-dependent end joining in G1. Oncotarget 7, 57679-57693.

Baudat, F., Manova, K., Yuen, J.P., Jasin, M., and Keeney, S. (2000). Chromosome synapsis defects and sexually dimorphic meiotic progression in mice lacking Spo11. Mol Cell 6, 989-998.

Bosshard, S., Duroy, P.O., and Mermod, N. (2019). A role for alternative end-joining factors in homologous recombination and genome editing in Chinese hamster ovary cells. DNA Repair 82, 102691.

Chaudhuri, A.R., and Nussenzweig, A. (2017). The multifaceted roles of PARP1 in DNA repair and chromatin remodelling. Nat Rev Mol Cell Biol 18, 610-621.

Chicheportiche, A., Bernardino-Sgherri, J., de Massy, B., and Dutrillaux, B. (2007). Characterization of Spo11-dependent and independent phospho-H2AX foci during meiotic prophase I in the male mouse. J Cell Sci 120, 1733-1742.

de Murcia, J.M., Niedergang, C., Trucco, C., Ricoul, M., Dutrillaux, B., Mark, M., et al. (1997). Requirement of poly(ADPribose) polymerase in recovery from DNA damage in mice and in cells. Proc Natl Acad Sci U S A 94, 7303-7307.

de Rooij, D.G., and de Boer, P. (2003). Specific arrests of spermatogenesis in genetically modified and mutant mice. Cytogenet Genome Res 103, 267-276.

Derijck, A., van der Heijden, G., Giele, M., Philippens, M., and de Boer, P. (2008). DNA double-strand break repair in parental chromatin of mouse zygotes, the first cell cycle as an origin of de novo mutation. Human Mol Genet 17, 1922-1937.

Di Meglio, S., Denegri, M., Vallefuoco, S., Tramontano, F., Scovassi, A.I., and Quesada, P. (2003). Poly(ADPR) polymerase-1 and poly(ADPR) glycohydrolase level and distribution in differentiating rat germinal cells. Mol Cell Biochem 248, 85-91.

Flohr, C., Bürkle, A., Radicella, J.P., and Epe, B. (2003). Poly(ADP-ribosyl)ation accelerates DNA repair in a pathway dependent on Cockayne syndrome B protein. Nucleic Acids Res 31, 5332-5337.

Fukushima, T., Takata, M., Morrison, C., Araki, R., Fujimori, A., Abe, M., et al. (2001). Genetic analysis of the DNAdependent protein kinase reveals an inhibitory role of $\mathrm{Ku}$ in late S-G2 phase DNA double-strand break repair. J Biol Chem 276, 44413-44418.

Gerton, J.L., and Hawley, S. (2005). Homologous chromosome interactions in meiosis: Diversity amidst conservation. Nat Rev Genet 6, 477-487.

Hamer, G., Roepers-Gajadien, H.L., van Duyn-Goedhart, A., Gademan, I.S., Kal, H.B., van Buul, P.P., et al. (2003). Function of DNA-protein kinase catalytic subunit during the early meiotic prophase without Ku70 and Ku86. Biol Reprod 68, 717-721.

Hunter, N. (2015). Meiotic recombination: the essence of heredity. Cold Spring Harb Perspect Biol 7.

Iliakis, G., Wang, H., Perrault, A.R., Boecker, W., Rosidi, B., Windhofer, F., et al. (2004). Mechanisms of DNA double strand break repair and chromosome aberration formation. Cytogenet Genome Res 104, 14-20.

Kakarougkas, A., and Jeggo, P.A. (2014). DNA DSB repair pathway choice: an orchestrated handover mechanism. Br J Radiol 87, 20130685. 
Kang, Y.J., and Yan, C.T. (2018). Regulation of DNA repair in the absence of classical non-homologous end joining. DNA Repair 68, 34-40.

Keeney, S., Giroux, C.N., and Kleckner, N. (1997). Meiosisspecific DNA double-strand breaks are catalyzed by Spo11, a member of a widely conserved protein family. Cell 88, 375384.

Liebe, B., Petukhova, G., Barchi, M., Bellani, M., Braselmann, H., Nakano, T., et al. (2006). Mutations that affect meiosis in male mice influence the dynamics of the mid-preleptotene and bouquet stages. Exp Cell Res 312, 3768-3781.

Lobrich, M., Shibata, A., Beucher, A., Fisher, A., Ensminger, M., Goodarzi, A.A., et al. (2010). gammaH2AX foci analysis for monitoring DNA double-strand break repair: strengths, limitations and optimization. Cell Cycle 9, 662-669.

Luijsterburg, M.S., de Krijger, I., Wiegant, W.W., Shah, R.G., Smeenk, G., de Groot AJL, et al. (2016). PARP1 Links CHD2-Mediated Chromatin Expansion and H3.3 Deposition to DNA Repair by Non-homologous End-Joining. Mol Cell 61, 547-562.

Ma, W., Halweg, C.J., Menendez, D., and Resnick, M.A. (2012). Differential effects of poly(ADP-ribose) polymerase inhibition on DNA break repair in human cells are revealed with Epstein-Barr virus. Proc Natl Acad Sci U S A 109, 6590-6595.

Mahadevaiah, S.K., Turner, J.M., Baudat, F., Rogakou, E.P., de Boer, P., Blanco-Rodriguez, J., et al. (2001). Recombinational DNA double-strand breaks in mice precede synapsis. Nat Genet 27, 271-276.

Mansour, W.Y., Borgmann, K., Petersen, C., Dikomey, E., and Dahm-Daphi, J. (2013). The absence of Ku but not defects in classical non-homologous end-joining is required to trigger PARP1-dependent end-joining. DNA Repair 12, 1134-1142.

Maymon, B.B., Cohen-Armon, M., Yavetz, H., Yogev, L., Lifschitz-Mercer, B., Kleiman, S.E., et al. (2006). Role of poly(ADP-ribosyl)ation during human spermatogenesis. Fertil Steril 86, 1402-1407.

Muhtadi, R., Ahmed, E.A., and Scherthan, H. (2017). Spermatocyte spreading during meiotic cell preparation is a two step process. J Cytol Histol 8, 1000450.

Pazzaglia, S., and Pioli, C. (2020). Multifaceted role of PARP-1 in DNA repair and inflammation: pathological and therapeutic implications in cancer and non-cancer diseases. Cells 9, 41.

Peters, A.H., Plug, A.W., van Vugt, and de Boer, P.M.J. (1997). A drying-down technique for the spreading of mammalian meiocytes from the male and female germline. Chromosome Res 5, 66-68.

Rogakou, E.P., Pilch, D.R., Orr, A.H., Ivanova, V.S., and Bonner, W.M. (1998). DNA double-stranded breaks induce histone H2AX phosphorylation on serine 139. J Biol Chem 273, 5858-5868. doi: 10.1074/jbc.273.10.5858.

Rogakou, P., Boon, C., Redon, C., and Bonner, W.M. (1999). Megabase chromatin domains involved in DNA doublestrand breaks in vivo. J Cell Biol 146, 905-916.
Rothkamm, K., Barnard, S., Moquet, J., Ellender, M., Rana, Z., and Burdak-Rothkamm, S. (2015). DNA damage foci: meaning and significance. Environ Mol Mutagen 56, 491-504.

Scherthan, H., Hieber, L., Braselmann, H., Meineke, V., and Zitzelsberger, H. (2008). Accumulation of DSBs in gammaH2AX domains fuel chromosomal aberrations. Biochem Biophys Res Commun 371, 694-697.

Shall, S., and de Murcia, G. (2000). Poly(ADP-ribose) polymerase-1: what have we learned from the deficient mouse model? Mutat Res/DNA Repair 460, 1-15.

Singh, P., Aggarwal, L.M., Parry, S.A., and Raman, M.J. (2018). Radiation dosimetry and repair kinetics of DNA damage foci in mouse pachytene spermatocyte and round spermatid stages. Mutagenesis 33, 231-239.

Slade, D. (2019). Mitotic functions of poly(ADP-ribose) polymerases. Biochem Pharmacol 167, 33-43.

Soni, A., Siemann, M., Grabos, M., Murmann, T., Pantelias, G.E., and Iliakis, G. (2014). Requirement for Parp-1 and DNA ligases 1 or 3 but not of Xrcc1 in chromosomal translocation formation by backup end joining. Nucleic Acids Res 42, 6380-6392.

Thomas, C., and Tulin, A.V. (2013). Poly-ADP-ribose polymerase: machinery for nuclear processes. Mol Aspects Med 34, 1124-1137.

Tramontano, F., Malanga, M., and Quesada, P. (2007). Differential contribution of poly(ADP-ribose)polymerase-1 and -2 (PARP-1 and -2) to the poly(ADP-ribosyl)ation reaction in rat primary spermatocytes. Mol Human Reprod 13, 821-828.

Wang, Z.Q., Stingl, L., Morrison, C., Jantsch, M., Los, M., Schulze-Osthoff, K., et al. (1997). PARP is important for genomic stability but dispensable in apoptosis. Genes Dev 11, 2347-2358.

Yang, F., Baumann, C., and De La Fuente, R. (2009). Persistence of histone $\mathrm{H} 2 \mathrm{AX}$ phosphorylation after meiotic chromosome synapsis and abnormal centromere cohesion in poly (ADP-ribose) polymerase (Parp-1) null oocytes. Dev Biol 331, 326-338.

Zickler, D., and Kleckner, N. (2015). Recombination, pairing, and synapsis of homologs during meiosis. Cold Spring Harb Perspect Biol 7.

Address correspondence to: Emad A. Ahmed, PhD Biological Sciences Department College of Science King Faisal University Al-Ahsa 31982 Saudi Arabia

E-mail: eaahmed@kfu.edu.sa

Received for publication October 22, 2020; received in revised form October 22, 2020; accepted October 29, 2020. 